\title{
HIGHER ELLIPTIC GENERA
}

\author{
Lev Borisov and Anatoly Libgober
}

\begin{abstract}
We show that elliptic classes introduced in [7] for spaces with infinite fundamental groups yield Novikov's type higher elliptic genera which are invariants of Kequivalence. This include, as a special case, the birational invariance of higher Todd classes studied recently by J.Rosenberg and J.Block-S.Weinberger. We also prove the modular properties of these genera, show that they satisfy a McKay correspondence, and consider their twist by discrete torsion.
\end{abstract}

\section{Introduction and statements of results.}

In works [26] and [3] the authors considered the birational properties of higher Todd, $L$ and $\hat{A}$ "genera" for nonsingular algebraic varieties. These "genera" are originated in the Novikov's conjecture that for a compact closed manifold $X$ with a fundamental group $\pi$ the higher signatures $\sigma_{\alpha}:\left(L(X) \cup f^{*}(\alpha)\right)[X]$ are homotopy invariants. Here $L(X) \in H^{*}(X, \mathbf{Q})$ is the total $L$-class, $f$ is the map from $X$ to the classifying space $B \pi$ of the group $\pi, \alpha \in H^{*}(B \pi, \mathbf{Q})$ and $[X]$ is the fundamental class of $X$ (cf. [13] for a survey of Novikov's conjecture). The work [26] conjectures that in the case when $X$ is projective algebraic and nonsingular the higher Todd invariants $\left(T d(X) \cup f^{*}(\alpha)\right)[X]$, where $T d$ is the total Todd class, are birational invariants. The work [3] contains a proof of this conjecture but also raises the problem of generalizing it to higher elliptic genera.

The purpose of this note is to present such a generalization for the (two-variable) elliptic genus. In fact, this generalization is done here in a wider category consisting of Kawamata log-terminal pairs $(X, D)$ for which $X$ supports the action of a finite group $G$ so that the pair $(X, D)$ is $G$-normal (cf. below). In particular we obtain the invariance of elliptic genus for $K$-equivalences, which, due to the fact that the Todd genus is a specialization of the elliptic genus, contains the birational invariance of higher Todd genus as a special case. We show also that many properties of ordinary elliptic genus (indicated in the abstract above) also are satisfied by the higher elliptic genera. These two variable invariants extend one variable higher elliptic genera considered (in smooth case) in connection with rigidity properties in [24].

Let $X$ be a complex manifold. The (two variable) elliptic genus is defined as the holomorphic Euler characteristic of

$$
y^{-\frac{d i m X}{2}} \otimes_{n \geq 1}\left(\Lambda_{-y q^{n-1}} T_{X}^{*} \otimes \Lambda_{-y^{-1} q^{n}} T_{X} \otimes S_{q^{n}} T_{M}^{*} \otimes S_{q^{n}} T_{M}\right)
$$

where $T_{X}$ is the tangent bundle, $T_{X}^{*}$ is its dual and for a bundle $V$, the element $S_{t} V$ (resp. $\Lambda_{t} V$ ) is the power series over the semigroup of vector bundles on $X$

Received by the editors May 18, 2006. 
given by $\sum_{k \geq 0} t^{k} S^{k}(V)$ (resp. $\sum_{k \geq 0} t^{k} \Lambda^{k}(V)$ ). One views this holomorphic Euler characteristic as a function on $\mathbf{C} \times H$ where $H$ is the upper half plane using $y=$ $\exp (2 \pi i z), q=\exp (2 \pi i \tau), z \in \mathbf{C}, \tau \in H$. As such, the elliptic genus becomes a holomorphic function on $\mathbf{C} \times H$. Considering the limit of this function when $\Im z, \Im \tau \rightarrow$ $\infty$ one recovers the Todd genus of $X$, as is seen directly from the definition. Elliptic genus has the $L$ and $\hat{A}$ genera as certain limit values as well (cf. [5]).

It follows from the Riemann-Roch theorem and a direct calculation (cf. [5]) that the elliptic genus can be written in terms the Chern roots $x_{i}$ of the tangent bundle of $X$ as $\mathcal{E} \mathcal{L} \mathcal{L}(X)[X]$ where

$$
\mathcal{E} \mathcal{L} \mathcal{L}(X)=\prod_{i} x_{i} \frac{\theta\left(\frac{x_{i}}{2 \pi \mathrm{i}}-z, \tau\right)}{\theta\left(\frac{x_{i}}{2 \pi \mathrm{i}}, \tau\right)}
$$

and

$$
\theta(z, \tau)=q^{\frac{1}{8}}(2 \sin \pi z) \prod_{l=1}^{l=\infty}\left(1-q^{l}\right) \prod_{l=1}^{l=\infty}\left(1-q^{l} \mathrm{e}^{2 \pi \mathrm{i} z}\right)\left(1-q^{l} \mathrm{e}^{-2 \pi \mathrm{i} z}\right)
$$

is the classical theta function considered as the series in $y, q$ (cf. [11]).

Now let $X$ be a complex manifold as above and let $\pi$ be its fundamental group. Let $f: X \rightarrow B \pi$ be the corresponding map and let $\alpha \in H^{k}(\pi, \mathbf{Q})$.

Definition 1.1. The higher elliptic genus is

$$
\operatorname{Ell}_{\alpha}(X)=\left(\mathcal{E} \mathcal{L} \mathcal{L}(X) \cup f^{*}(\alpha)\right)[X]
$$

where the elliptic class $\mathcal{E} \mathcal{L} \mathcal{L}(X) \in H^{*}(X, \mathbf{Q})$ is given by (2).

Modular property of $E l l_{\alpha}(X)$ is described in theorem 1.5 below but first we shall outline the extension to non simply-connected case of the generalizations of elliptic genus introduced in [6] and [7].

Let $X$ be a normal projective algebraic variety and $D=\sum a_{i} D_{i}$ be a linear combination of distinct irreducible divisors with rational coefficients. The pair $(X, D)$ is called Kawamata log-terminal (cf. [25]) if $K_{X}+D$ is Q-Cartier and there is a birational morphism $f: Y \rightarrow X$ such that the union of the proper preimages of components of $D$ and the components of exceptional set $E=\cup E_{j}$ form a normal crossing divisor such that $K_{Y}=f^{*}\left(K_{X}+\sum a_{i} D_{i}\right)+\sum \alpha_{j} E_{j}$ and $\alpha_{j}>-1$ (here $K_{X}, K_{Y}$ are the canonical classes of $X$ and $Y$ respectively). The triple $(X, D, G)$ where $X$ is a nonsingular variety, $D$ is a divisor and $G$ is a finite group of biholomorphic automorphisms is called $G$-normal (cf. [4], [7]) if the components of $D$ form a normal crossings divisor and the isotropy group of any point acts trivially on the components of $D$ containing this point.

Definition 1.2. (cf. [7] definition 3.2) Let $(X, D)$ be a Kawamata log terminal $G$ normal pair and $D=-\sum \delta_{k} D_{k}$. The orbifold elliptic class of $(X, D, G)$ is the class in $H_{*}(X, \mathbf{Q})$ given by:

$$
\mathcal{E} \mathcal{L} \mathcal{L}(X, D, G ; z, \tau):=\frac{1}{|G|} \sum_{g, h, g h=h g} \sum_{X^{g, h}}\left[X^{g, h}\right]\left(\prod_{\lambda(g)=\lambda(h)=0} x_{\lambda}\right)
$$




$$
\begin{gathered}
\times \prod_{\lambda} \frac{\theta\left(\frac{x_{\lambda}}{2 \pi \mathrm{i}}+\lambda(g)-\tau \lambda(h)-z\right)}{\theta\left(\frac{x_{\lambda}}{2 \pi \mathrm{i}}+\lambda(g)-\tau \lambda(h)\right)} \mathrm{e}^{2 \pi \mathrm{i} \lambda(h) z} \\
\times \prod_{k} \frac{\theta\left(\frac{e_{k}}{2 \pi \mathrm{i}}+\epsilon_{k}(g)-\epsilon_{k}(h) \tau-\left(\delta_{k}+1\right) z\right)}{\theta\left(\frac{e_{k}}{2 \pi \mathrm{i}}+\epsilon_{k}(g)-\epsilon_{k}(h) \tau-z\right)} \frac{\theta(-z)}{\theta\left(-\left(\delta_{k}+1\right) z\right)} \mathrm{e}^{2 \pi \mathrm{i} \delta_{k} \epsilon_{k}(h) z} .
\end{gathered}
$$

where in definition $1.2 X^{g, h}$ denotes an irreducible component of the fixed set of the commuting elements $g$ and $h$ and $\left[X^{g, h}\right]$ denotes the image of the fundamental class in $H_{*}(X)$. The restriction of $T X$ to $X^{g, h}$ splits into linearized bundles according to the $\left([0,1)\right.$-valued) characters $\lambda$ of $\langle g, h\rangle$. Moreover, $e_{k}=c_{1}\left(E_{k}\right)$ and $\epsilon_{k}$ is the character of $\mathcal{O}\left(E_{k}\right)$ restricted to $X^{g, h}$ if $E_{k}$ contains $X^{g, h}$ and is zero otherwise.

Definition 1.3. Let $\pi$ and $\alpha$ be as in definition 1.1 and other notations as in the definition 1.2. Then

$$
E l_{\alpha}(X, D, G)=\left(\mathcal{E} \mathcal{L} \mathcal{L}(X, D, G) \cap f^{*}(\alpha)\right)_{0}
$$

i.e. the component of degree zero of the class in $H_{*}(X, \mathbf{Q})$.

Special cases of the elliptic class $\mathcal{E} \mathcal{L} \mathcal{L}(X, D, G)$ are the following.

a) If $G$ is the trivial group one obtains higher elliptic genus of Kawamata log terminal pairs $\mathcal{E} \mathcal{L} \mathcal{L}(X, D)$. If $D=\sum \delta_{k} D_{k}$ and $d_{k} \in H^{2}(X, \mathbf{Q})$ is the cohomology class dual to $D_{k}$ then:

$$
\mathcal{E} \mathcal{L} \mathcal{L}(X, D)=\left(\prod_{l} \frac{\left(\frac{x_{l}}{2 \pi \mathrm{i}}\right) \theta\left(\frac{x_{l}}{2 \pi \mathrm{i}}-z\right) \theta^{\prime}(0)}{\theta(-z) \theta\left(\frac{x_{l}}{2 \pi \mathrm{i}}\right)}\right) \times\left(\prod_{k} \frac{\theta\left(\frac{d_{k}}{2 \pi \mathrm{i}}-\left(\delta_{k}+1\right) z\right) \theta(-z)}{\theta\left(\frac{d_{k}}{2 \pi \mathrm{i}}-z\right) \theta\left(-\left(\delta_{k}+1\right) z\right)}\right)
$$

b) If $D=\emptyset$ one obtains the higher orbifold elliptic genus $E l l_{o r b, \alpha}$ which is the value on $[X]$ of the cup product with $f^{*}(\alpha)$ of the orbifold elliptic class:

$$
\begin{aligned}
& \mathcal{E} \mathcal{L} \mathcal{L}_{\text {orb }}(X, G)= \\
& \frac{1}{|G|} \sum_{g, h, g h=h g}\left(\prod_{\lambda(g)=\lambda(h)=0} x_{\lambda}\right) \prod_{\lambda} \frac{\theta\left(\tau, \frac{x_{\lambda}}{2 \pi \mathrm{i}}+\lambda(g)-\tau \lambda(h)-z\right)}{\theta\left(\tau, \frac{x_{\lambda}}{2 \pi \mathrm{i}}+\lambda(g)-\tau \lambda(h)\right)} e^{2 \pi \mathrm{i} \lambda(h) z}\left[X^{g, h}\right]
\end{aligned}
$$

Subscript $\alpha$ will denote the twisting by the class $\alpha$ of the genus corresponding to (4) and (5). Either of these is a special case of 1.3.

In the case $D=\emptyset, G=\{1\}$ we obtain the class given by (2) and the higher elliptic genus defined in 1.1 .

Finally recall the following (cf. [16])

Definition 1.4. A Jacobi form of index $t \in \frac{1}{2} \mathbf{Z}$ and weight $k$ is a holomorphic function $\chi$ on $H \times \mathbf{C}$ satisfying the following functional equations:

$$
\begin{gathered}
\chi\left(\frac{a \tau+b}{c \tau+d}, \frac{z}{c \tau+d}\right)=(c \tau+d)^{k} e^{\frac{2 \pi i t c z^{2}}{c \tau+d}} \chi(\tau, z) \\
\chi(\tau, z+\lambda \tau+\mu)=(-1)^{2 t(\lambda+\mu)} e^{-2 \pi i t\left(\lambda^{2} \tau+2 \lambda z\right)} \chi(\tau, z)
\end{gathered}
$$

Important property of the elliptic genus is that for a $S U$-manifold the elliptic genus is a Jacobi form having weight zero and index $\frac{\operatorname{dim} X}{2}$. 
Theorem 1.5. Let $X$ be a SU-manifold, $d=\operatorname{dim} X, \pi=\pi_{1}(X)$ and $\alpha \in H^{2 k}(\pi, \mathbf{Q})$. Then the higher elliptic genus $\left(\mathcal{E} \mathcal{L} \mathcal{L}(X) \cup f^{*}(\alpha)\right)[X]$ is a Jacobi form having index $\frac{d}{2}$ and weight $-k$. It has the Novikov signature, the higher Todd genus and higher $\hat{A}$-genus as specializations.

More generally, let $X, D$ be a Kawamata log-terminal $G$-normal pair where $G$ is a finite group. If $m\left(K_{X}+D\right)$ is a trivial Cartier divisor, $n$ is the order of the image $G \rightarrow$ Aut $H^{0}\left(X, m\left(K_{X}+D\right)\right)$ and $\alpha \in H^{2 k}\left(\pi_{1}(X), \mathbf{Q}\right)$ as above then $\mathcal{E} \mathcal{L} \mathcal{L}_{\alpha}(X, D, G, z, \tau)$ is a Jacobi form having weight $-k$ and the index $\frac{\operatorname{dim} X}{2}$ for the subgroup of Jacobi group generated by:

$$
(z, \tau) \rightarrow(z+m n, \tau),(z, \tau) \rightarrow(z+m n \tau),(z, \tau) \rightarrow(z, \tau),(z, \tau) \rightarrow\left(\frac{z}{\tau},-\frac{1}{\tau}\right)
$$

Next recall that two manifolds $X_{1}, X_{2}$ are called $K$-equivalent if there is a smooth manifold $\tilde{X}$ and a diagram:

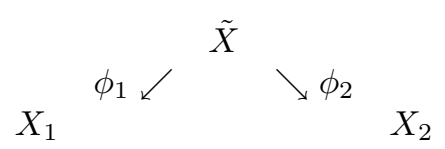

in which $\phi_{1}$ and $\phi_{2}$ are birational morphisms and $\phi_{1}^{*}\left(K_{X_{1}}\right)$ and $\phi_{2}^{*}\left(K_{X_{2}}\right)$ are linearly equivalent.

Theorem 1.6. For any $\alpha \in H^{*}(B \pi, \mathbf{Q})$ the higher elliptic genus

$$
\left(\mathcal{E} \mathcal{L} \mathcal{L}(X) \cup f^{*}(\alpha),[X]\right)
$$

is an invariant of $K$-equivalence. Moreover, if $(X, D, G)$ and $(\hat{X}, \hat{D}, G)$ are $G$-normal and Kawamata log-terminal and if $\phi:(\hat{X}, \hat{D}) \rightarrow(X, D)$ is $G$-equivariant such that

$$
\phi^{*}\left(K_{X}+D\right)=K_{\hat{X}}+\hat{D}
$$

then

$$
E l l_{\alpha}(\hat{X}, \hat{D}, G)=E l l_{\alpha}(X, D, G)
$$

In particular the higher elliptic genera (and hence the higher signatures and $\hat{A}$-genus) are invariant for crepant birational morphisms and the specialization into the Todd class is birationally invariant.

Remark 1.7. Since the fundamental groups are rather restricted by algebraic geometry, one may wonder when higher genera yield new invariants. This also depends on the existence of nontrivial cohomology classes of the fundamental group (cf. remark 2.1 below). By a result of Beauville a fundamental group of a Calabi Yau manifold is an extension of a free abelian group by a finite group (cf. [2]) so one does obtain new invariants if the rank of this abelian group is positive. On the other hand, the higher elliptic genera of pairs are defined with $X$ being arbitrary projective algebraic manifold and class of groups from which one obtains higher invariants is much bigger than in Calabi Yau case. 


\section{Proofs}

Proof of the theorem 1.5. The argument is essentially the same as for the special $(k=0)$ case in [5] or in more general case of orbifold elliptic genus of pairs dealt with in [7]. We spell out the argument only in the first case of the theorem 1.5. It is enough to check the transformation formulas on the generators of the Jacobi groups i.e. that

$$
\begin{gathered}
\chi(M, z, \tau+1)=\chi(M, z, \tau) \\
\chi\left(M, \frac{z}{\tau},-\frac{1}{\tau}\right)=\tau^{k} \mathrm{e}^{\frac{\pi \mathrm{i} d z^{2}}{\tau}} \chi(M, z, \tau) \\
\chi(M, z+\tau, \tau)=(-1)^{d} \mathrm{e}^{-\pi \mathrm{i} d(\tau+2 z)} \chi(M, z, \tau) \\
\chi(M, z+1, \tau)=(-1)^{d} \chi(M, z, \tau) .
\end{gathered}
$$

Let

$$
\prod x_{i} \frac{\theta\left(\frac{x_{i}}{2 \pi \mathrm{i}}-z, \tau\right)}{\theta\left(\frac{x_{i}}{2 \pi \mathrm{i}}, \tau\right)}=\sum_{\mathbf{k}} Q_{\mathbf{k}}(z, \tau) \mathbf{x}^{\mathbf{k}}
$$

where $\mathbf{x}$ is a product of powers of $x_{i}$ and $\mathbf{k}$ is multiindex. The higher elliptic genus is the linear combination of functions $Q_{\mathbf{k}}(z, \tau)$ with coefficients $f^{*}(\alpha) \cup \mathbf{x}^{\mathbf{k}}[X]$ for all $\mathbf{k},|\mathbf{k}|=d-k$. The transformation formulas for $\theta$-function yield that the left hand side satisfies (7),(8) and (9). Hence these relations are valid for all $Q_{\mathbf{k}}(z, \tau)$. For the transformations $\tau \rightarrow-\frac{1}{\tau}, x_{i} \rightarrow \frac{x_{i}}{\tau}, z \rightarrow \frac{z}{\tau}$ we have:

$$
\begin{aligned}
& \sum_{\mathbf{k}} Q_{\mathbf{k}}\left(\frac{z}{\tau},-\frac{1}{\tau}\right)\left(\frac{\mathbf{x}}{\tau}\right)^{\mathbf{k}}= \\
& \prod_{i}\left(\frac{x_{i}}{\tau}\right) \frac{\theta\left(-\frac{z}{\tau}+\frac{x_{i}}{2 \pi \mathrm{i} \tau},-\frac{1}{\tau}\right)}{\theta\left(\frac{x_{i}}{2 \pi \mathrm{i} \tau},-\frac{1}{\tau}\right)}=\left(\frac{1}{\tau}\right)^{d} \prod \mathrm{e}^{\frac{-z x_{i}}{\tau}} x_{i} \frac{\mathrm{e}^{\frac{\pi \mathrm{i} z^{2}}{\tau}} \theta\left(-z+\frac{x_{i}}{2 \pi \mathrm{i}}, \tau\right)}{\theta\left(\frac{x_{i}}{2 \pi \mathrm{i}}, \tau\right)} \\
& =\left(\frac{1}{\tau}\right)^{d} \prod x_{i} \frac{\mathrm{e}^{\frac{\pi \mathrm{i} z^{2}}{\tau}} \theta\left(-z+\frac{x_{i}}{2 \pi \mathrm{i}}, \tau\right)}{\theta\left(\frac{x_{i}}{2 \pi \mathrm{i}}, \tau\right)}=\sum_{\mathbf{k}}\left(\frac{1}{\tau}\right)^{d} \mathrm{e}^{\frac{\pi \mathrm{i} \mathrm{d} z^{2}}{\tau}} Q_{\mathbf{k}}(z, \tau) \mathbf{x}^{\mathbf{k}}
\end{aligned}
$$

(the equality before the last is due to the vanishing of $c_{1}$ ). Hence the coefficient of a monomial $\mathbf{x}^{\mathbf{k}}$ with $|\mathbf{k}|=d-k$ satisfies:

$$
Q_{\mathbf{k}}\left(\frac{z}{\tau},-\frac{1}{\tau}\right)=t^{-k} \mathrm{e}^{\frac{\pi \mathrm{i} d z^{2}}{\tau}} Q_{\mathbf{k}}(z, \tau)
$$

Remark 2.1. Elliptic genus $\operatorname{Ell}_{\alpha}(X)=0$ if $\alpha \in H^{2 k}\left(\pi_{1}, \mathbf{Q}\right), k>d$ and is a multiple of the Jacobi form:

$$
\left(\frac{\theta(\tau, z)}{\theta^{\prime}(0, \tau)}\right)^{d}
$$

for $\alpha \in H^{2 d}\left(\pi_{1}, \mathbf{Q}\right)$. The latter has weight $-d$ and index $\frac{d}{2}$. 
For a class $\alpha \in H^{2 d-2}\left(\pi_{1}(X), \mathbf{Q}\right)$ the genus Ell ${ }_{\alpha}(X)$ is determined by the higher Todd genus but elliptic genera corresponding to classes of large codimension cannot be described in terms of higher Todd (or $\chi_{y}$ )-genus (cf. [5], theorem 2.7).

Proof of the theorem 1.6. We apply Theorem 3.5 of [7] to the resolution $\phi_{1}: \tilde{X} \rightarrow X_{1}$ of the diagram (6) to get a direct image formula

$$
\left(\phi_{1}\right)_{*} \mathcal{E} \mathcal{L} \mathcal{L}(\tilde{X}, \tilde{D}, G ; z, \tau)=\mathcal{E} \mathcal{L} \mathcal{L}\left(X_{1}, D_{1}, G ; z, \tau\right) .
$$

Here $\tilde{D}$ is defined as usual as having the same coefficients as $D_{1}$ at components of the proper preimage of $D_{1}$ and having the coefficients at the exceptional divisors of $\phi_{1}$ determined from $\phi^{*}\left(K_{X}+D\right)=K_{\hat{X}}+\tilde{D}$.

Together with the diagram

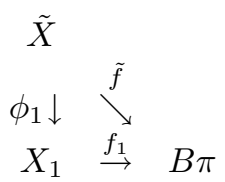

the direct image formula yields

$$
\begin{gathered}
\left(\mathcal{E} \mathcal{L} \mathcal{L}_{\alpha}(\tilde{X}, \tilde{D}, G) \cap \tilde{f}^{*}(\alpha)\right)_{0}= \\
\left.\left(\phi_{1}\right)_{*}\left(\mathcal{E} \mathcal{L} \mathcal{L}(\tilde{X}, \tilde{D}, G) \cap \hat{(} \phi_{1} \circ f_{1}\right)^{*}(\alpha)\right)_{0}=\left(\mathcal{E} \mathcal{L} \mathcal{L}\left(X_{1}, D_{1}, G\right) \cap f_{1}^{*}(\alpha)\right)_{0} .
\end{gathered}
$$

The $K$-equivalence means that the divisor $\tilde{D}$ calculated for $\phi_{1}$ is the same as the divisor $\tilde{D}$ calculated for $\phi_{2}$, which shows that higher elliptic genera are invariant under $K$-equivalences. The claim about crepant morphisms is then immediate. Finally, as one sees from formula (4), in the limit $\Im z \rightarrow \infty, \tau \rightarrow \infty$ the elliptic class of pair loses its dependence on $D$. Hence the push forward formula is valid without assumption on the canonical class and the higher Todd classes are invariant for arbitrary birational morphisms and not just $K$-equivalences.

\section{Further properties of higher genera}

3.1. Higher elliptic genera of singular varieties. One of the consequence of previous discussion is existence of well defined higher elliptic genera of projective algebraic varieties with log-terminal singularities.

If $\tilde{X}$ is a resolution of a projective variety $X$ then $\pi_{1}(X) \neq \pi_{1}(\tilde{X})$ in general (for example image of generic projection of $X$ in $\mathbf{P}^{\operatorname{dim} X+1}$ is simply-connected for any $X$ (cf. [17])). However we have the following result due to Takayama (cf. [27](*)):

Lemma 3.1. Assume that $X$ has only log-terminal singularities (or more generally $(X, \Delta)$ has divisorial Kawamata log-terminal singularities in terminology of [20]) and let $f: X^{\prime} \rightarrow X$ be a resolution of singularities of $X$. Then $\pi_{1}\left(X^{\prime}\right)=\pi_{1}(X)$.

It follows from the theorem 1.6 that the following definition yields result independent of resolution.

$\left({ }^{*}\right)$ We thank C.Hacon and J.McKernan for pointing out the reference and J.McKernan for further comments. 
Definition 3.2. Let $X$ be a projective algebraic variety with Q-Gorenstein log-terminal singularities. Let $\alpha \in H^{*}\left(\pi_{1}(X), \mathbf{Q}\right)$ be the cohomology class of its fundamental group. If $\phi: \tilde{X} \rightarrow X$ is a resolution of singularities of $X, K_{\tilde{X}}=\phi^{*}\left(K_{X}\right)+\tilde{D}$ and $\alpha$ is viewed as the element in the $H^{*}\left(\pi_{1}(\tilde{X}), \mathbf{Q}\right) \stackrel{\text { dfn }}{=} H^{*}\left(\pi_{1}(X), \mathbf{Q}\right)$ then:

$$
E l l_{\alpha}(X)=E l l_{\alpha}(\tilde{X}, \tilde{D})
$$

Note that similarly one can define the $\operatorname{Ell}_{\alpha}(X, D)$ where $(X, D)$ is such that $K_{X}+D$ is $\mathbf{Q}$-Cartier and such that $(X, D)$ is a log-terminal pair. A large class of varieties with singularities as in this definition can be obtained by looking at the quotients of nonsingular varieties by the action of a finite group acting via biholomorphic transformations. The theorem 3.3 of the next section shows that the calculation of the higher elliptic genus of quotients can be done in terms the action on nonsingular variety. This extends the results of [7] for ordinary elliptic genus which in turn extend the results on Euler characteristics, $\chi_{y}$-characteristic etc. of quotients (see this reference for review of the preceding work).

3.2. Higher McKay correspondence. Let $X$ be nonsingular and $G$, as before, a finite group of biholomorphic transformations. Let $\pi=\pi_{1}(X / G)$ and let $\alpha_{X / G} \in$ $H^{*}\left(\pi_{1}(X / G), \mathbf{Q}\right)$. Let $\mu_{G}: \pi_{1}(X) \rightarrow \pi_{1}(X / G)$ be the homomorphism corresponding to $X \rightarrow X / G, \alpha_{X}=\mu_{G}^{*}\left(\alpha_{X / G}\right) \in H^{*}\left(\pi_{1}(X), \mathbf{Q}\right)$. The next theorem describes the invariant $E l l_{o r b, \alpha}(X, G)$ given by class (5) in terms of resolution of singularities of $X / G$ (which is the classical McKay correspondence between the Euler characteristic of minimal resolution and the order of the group in the case $\pi_{1}=\{1\}, q=0, y=$ $\left.1, X=\mathbf{C}^{2}, G \subset S L_{2}(\mathbf{C})\right)$. In the case $\alpha=1$ the McKay correspondence for elliptic genera was conjectured in [6] and proven in [7]. In the case of arbitrary $\alpha$ we have the following.

Theorem 3.3. Let $X$ be a nonsingular projective variety, $G$ acts biholomorphically on $X, \mu: X \rightarrow X / G, D=\sum\left(\nu_{i}-1\right) D_{i}$ is the ramification divisor of $\mu$ and $\Delta_{X / G}=$ $\sum \frac{\nu_{i}-1}{\nu_{i}} \mu\left(D_{i}\right)$. Then:

$$
E l l_{o r b, \mu^{*}\left(\alpha_{X} / G\right)}(X, G ; z, \tau)=E l l_{\alpha_{X / G}}\left(X / G, \Delta_{X / G} ; z, \tau\right)
$$

Proof. Indeed, this follows by the same argument as the one used in the proof of the theorem 5.3 in [7] by applying (obtained in lemma 5.4, [7]) the push forward formulas and the projection formula to the diagram:

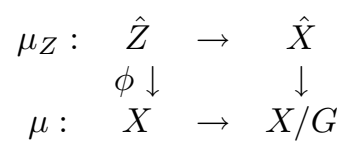

since both sides of the claimed equality are pushforwards of the same class $\mathcal{E} \mathcal{L} \mathcal{L}(\hat{Z}, \hat{D}, G)$ on $\hat{Z}$. 
3.3. Concluding remarks: flops, rigidity and discrete torsion. Higher elliptic genera yield a description of $\Omega^{S U}(B \pi) \otimes \mathbf{Q}$ modulo flops using Jacobi forms extending Totaro's (cf.[28]) description in the case $\pi=1$.

Definition 3.4. We shall say that two maps $f_{X}: X \rightarrow B \pi$ and $f_{X^{\prime}}: X^{\prime} \rightarrow B \pi$ are differ by a flop if:

a) there is a map of an (almost) complex space $f_{Y}: Y \rightarrow B \pi$ such that the singular set $\operatorname{Sing} Y=Z$ is a manifold having in $Y$ a regular neighborhood biholomorphic to $Z \tilde{\times} \mathcal{V}$ where $\mathcal{V} \subset \mathbf{C}^{4}$ is a germ at the origin of the hypersurface given by $x y=z w$ and

b) there are maps $\pi_{X}: X \rightarrow Y$ and $\pi_{X^{\prime}}: X^{\prime} \rightarrow Y$ such that $\pi_{X}$ and $\pi_{X}^{\prime}$ are small resolutions of $Y$ (i.e. $\pi_{X}^{-1}(Z) \rightarrow Z$ and $\pi_{X^{\prime}}^{-1}(Z) \rightarrow Z$ are $\mathbf{P}^{1}$ fibrations yielding at each point of $Z$ two different $\mathbf{P}^{1}$-resolutions of the node $\mathcal{V}$ ) and such that one has the commutative diagram:

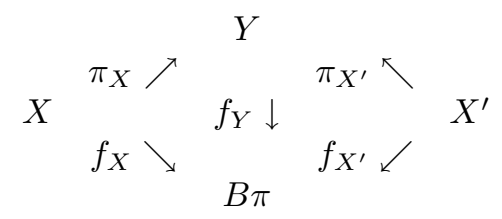

Proposition 3.5. Let $I_{\pi}$ (resp. I) be the ideal in $\Omega^{U}(B \pi)$ generated by the differences $\left(X, \pi_{X}\right)$ and $\left(X^{\prime}, \pi_{X^{\prime}}\right)$ from the above definition (resp. $X^{\prime}-X$ where $X^{\prime}$ and $X$ are differ by a classical flop (cf. [28]).) Then one has the following isomorphism of graded $\Omega_{*}^{U}$-modules:

$$
\Omega_{*}^{U}(B \pi) \otimes \mathbf{Q} / I_{\pi}=H_{*}(B \pi, \mathbf{Q}) \otimes_{\mathbf{Q}} \Omega_{*}^{U} / I=H_{*}(B \pi, \mathbf{Q}) \otimes \mathbf{Q}\left[x_{1}, x_{2}, x_{3}, x_{4}\right]
$$

(cf. [28] for geometric description of the isomorphism: $\Omega_{*}^{U} / I=\mathbf{Q}\left[x_{1}, x_{2}, x_{3}, x_{4}\right]$ ). In particular:

$$
\operatorname{Hom}\left(\Omega_{d}^{S U}(B \pi) /\left(I_{\pi} \cap \Omega_{d}^{S U}(B \pi)\right), \mathbf{Q}\right)=\oplus_{k \in \mathbf{Z}} H^{2 k}\left(B \pi, \mathrm{Jac}_{-k, \frac{d}{2}}\right)
$$

where $\mathrm{Jac}_{-k, \frac{d}{2}}$ is the space of Jacobi forms having weight $-k$ and index $\frac{d}{2}(*)$.

Proof. This follows from the isomorphism (cf.[12]):

$$
\oplus H_{k}(B \pi, \mathbf{Q}) \otimes \Omega_{d-k / 2}^{U} \otimes \mathbf{Q} \rightarrow \Omega_{d}^{U}(B \pi) \otimes \mathbf{Q}
$$

which assigns to an almost complex manifold $\pi_{N}: N \rightarrow B \pi$ representing a homology class $\alpha$ and an almost complex manifold $M$ the map of $M \times N$ which is the composition of the projection on $N$ and $\pi_{N}$. The condition that $X^{\prime}-X \in I$ is equivalent to $X^{\prime}-$ $X=F \tilde{\times} Z \rightarrow B \pi$ where $F$ is the almost complex $S U$-manifold which is homological $\mathbf{C P}^{3}$ (cf. [28]). The map (19) takes it to zero. Converse it similar. Note that for $\pi_{1}=(1)$ (18) becomes the identification in [28].

Let $X$ be a Calabi Yau algebraic manifold with the fundamental group $\pi$ and let $X \rightarrow \operatorname{Alb}(X)$ be the Albanese map. Since the fundamental group of $X$ has a free

$\left(^{*}\right)$ The isomorphism (18) is a counterpart of the theorem 4.3 in [26]. The assumption in [26] that $H_{k}(B \pi, \mathbf{Q})$ is spanned by classes of maps form projective varieties can be omitted if birational invariance is stated as triviality on the almost complex manifolds which are projectivisations of complex bundles: indeed any birational equivalence is composition of blowups and blowdowns (cf. [1]) and the difference of a manifold and its blow in $\Omega^{U}$ is a projectivised bundle (cf. [21]). 
abelian group of rank $2 \operatorname{dim} A l b(X)$ as a subgroup of finite index (cf. [2]) we have the following description of the higher ellptic genera which can appear in algebraic case. Using the isomorphism

$$
J_{-k, \frac{d}{2}}=\oplus J_{0, \frac{d-k}{2}}
$$

(which follows for example since the ring of Jacobi form has a system of generators such that only one generator $\left(\frac{\theta(\tau, z)}{\theta^{\prime}(0, \tau)}\right)$ has negative weight (cf. [19])) the isomorphism (19) implies that the higher genera coincide with the ordinary elliptic genera of preimages of (almost complex) submanifolds of the abelian variety $A l b(X)$ interpreted as elements of $J_{-k, \frac{d}{2}}$ using (20).

Other application of higher elliptic genera include:

a) Rigidity theorems extending the result of Browder-Hsiang (cf. [10]) on rigidity of higher $\hat{A}$-genus on one hand and Bott-Taubes results on rigidity of elliptic. Case of higher one variable elliptic genus is discussed in [24] and [15] (cf. also [29]).

b) One has a version of higher elliptic genera twisted by discrete torsion extending the one considered in [23]. Let $X, D$ be a $G$-normal pair where $G$ is a finite group of biholomorphic automorphisms. A class $\nu \in H^{2}(G, U(1))$ defines $\delta(g, h)=\frac{\alpha(g, h)}{\alpha(h, g)}$ which allows to twist using $\nu$ the elliptic class in definition 1.2 to obtains the class:

$$
\begin{gathered}
\mathcal{E} \mathcal{L}^{\nu}(X, D, G ; z, \tau):=\frac{1}{|G|} \sum_{g, h, g h=h g} \sum_{X^{g, h}}\left[X^{g, h}\right] \delta(g, h)\left(\prod_{\lambda(g)=\lambda(h)=0} x_{\lambda}\right) \\
\times \prod_{\lambda} \frac{\theta\left(\frac{x_{\lambda}}{2 \pi \mathrm{i}}+\lambda(g)-\tau \lambda(h)-z\right)}{\theta\left(\frac{x_{\lambda}}{2 \pi \mathrm{i}}+\lambda(g)-\tau \lambda(h)\right)} \mathrm{e}^{2 \pi \mathrm{i} \lambda(h) z} \\
\times \prod_{k} \frac{\theta\left(\frac{e_{k}}{2 \pi \mathrm{i}}+\epsilon_{k}(g)-\epsilon_{k}(h) \tau-\left(\delta_{k}+1\right) z\right)}{\theta\left(\frac{e_{k}}{2 \pi \mathrm{i}}+\epsilon_{k}(g)-\epsilon_{k}(h) \tau-z\right)} \frac{\theta(-z)}{\theta\left(-\left(\delta_{k}+1\right) z\right)} \mathrm{e}^{2 \pi \mathrm{i} \delta_{k} \epsilon_{k}(h) z} .
\end{gathered}
$$

This yields the following version of the elliptic genus:

$$
E l l_{\alpha}^{\nu}(X, D, G)=\left(\mathcal{E} \mathcal{L} \mathcal{L}^{\nu}(X, D, G) \cap f^{*}(\alpha)\right)_{0}
$$

This is a Jacobi form of weight $-k$ and index $\frac{d}{2}$. Such elliptic genus is also rigid for $S^{1}$ actions commuting with the action of $G$ and preserving $D$.

\section{Acknowledgements}

Both authors were partially supported by NSF grants.

\section{References}

[1] D. Abramovich, K. Karu, K. Matsuki, and J. Włodarczyk, Torification and Factorization of Birational Maps, J. Amer. Math. Soc. 15 (2002), no. 3, 531-572.

[2] A. Beauville, Variété Kahleriennes dont la premiere class Chern est nulle, J.Diff. Geom, 18 (1983), no.4, 755-782.

[3] J. Block and S. Weinberger, Higher Todd classes and holomorphic group actions. Pure Appl. Math. Q. 2 (2006), no. 4, 1237-1253.

[4] V. Batyrev, Non-Archimedean integrals and stringy Euler numbers of log-terminal pairs, J. Eur. Math. Soc. (JEMS) 1 (1999), no. 1, 5-33. 
[5] L. A. Borisov and A. Libgober, Elliptic Genera of Toric Varieties and Applications to Mirror Symmetry, Invent. Math. 140 (2000), no. 2, 453-485.

[6] _ Elliptic genera of singular varieties, Duke Math. J. 116 (2003), no. 2, 319-351.

[7] _ McKay correspondence for elliptic genera, Ann. of Math. (2) 161 (2005), no. 3, 15211569.

[8] R. Bott and C. Taubes, On the rigidity theorems of Witten. J. Amer. Math. Soc. 2 (1989), no. 1, 137-186.

[9] J. P. Brasselet, J. Schuermann, and S. Yokura, Hirzebruch classes and motivic Chern classes for singular spaces, math.AG/0503492.

[10] W. Browder and W. C. Hsiang, G-actions and the fundamental group, Inventiones Math. 65 (1981/82), no. 3, 411-424.

[11] K. Chandrasekharan, Elliptic functions, Fundamental Principles of Mathematical Sciences, 281, Springer-Verlag, Berlin-New York, 1985.

[12] P. Conner and E. Floyd, Differentiable Periodic Maps, Ergebnisse der Mathematic, band 33, Springer Verlag, 1964

[13] J. Davis, Manifold aspects of the Novikov conjecture, Surveys in surgery theory, vol.1, 195-224, Ann. of Math. Studies, Princeton Univ. Press. Princeton, N.J. 2000.

[14] C. Dong, K. Liu, and X. Ma, On orbifold elliptic genus, Contemp. Math., 310, Amer. Math. Soc., Providence, RI, 2002.

[15] D. Gong and K. Liu, Rigidity of higher elliptic genera, Ann. Global Anal.Geom. 14 (1996), 219-236.

[16] M. Eichler and D. Zagier, The theory of Jacobi forms, Progress in Mathematics, 55, Birkhuser Boston, Inc., Boston, Mass., 1985

[17] W. Fulton and R. Lazarsfeld, Connectctivity and its applications in algebraic geometry, Algebraic Geometry, (Chicago, Ill, 1980), pp.26-92. Lecture Notes in Math. Springer Verlag, 1981.

[18] M. Goresky and R. MacPherson, Stratified Morse Theory, Springer Verlag, 1988.

[19] V. Gritsenko, Complex vector bundles and Jacobi forms, Sriukaisekikenkyusho Kokyuroku No. 1103 (1999), 71-85.

[20] C. Hacon and J. McKernan, On Shokurov's Rational connectedness conjecture, Duke Math. J. 138 (2007), no. 1, 119-136.

[21] N. Hitchin, Harmonic Spinors, Advances in Math. 14 (1974), 1-55.

[22] J. Kollar, Y. Miyaoka, and S.Mori, Rationally connected varieties, J.Algebraic Geometry, 1 (1992), no. 3, 429-448.

[23] A. Libgober and M. Szczesny, Discrete torsion, orbifold elliptic genera, and the chiral de Rham complex, Pure Appl. Math. Q. 2 (2006), no. 4, 1217-1236.

[24] K. Liu, On Mod 2 and Higher Elliptic Genera, Comm. Math. Phys. 149 (1992), no. 1, 71-95.

[25] Y. Kawamata, K. Matsuda, and K. Matsuki, Introduction to the minimal model problem, Algebraic geometry, Sendai, 1985, 283-360, Adv. Stud. Pure Math., 10, North-Holland, Amsterdam, 1987.

[26] J. Rosenberg, An analog of Novikov's conjecture in complex algebraic geometry,preprint, math.AG/0509526.

[27] S. Takayama, Local simple connectedness of resolutions of log-terminal singularities, Internat. J. Math. 14 (2003), no. 8, 825-836.

[28] B. Totaro, Chern numbers of singular varieties and elliptic homology, Ann. of Math. 151 (2000), no. 2, 757-791.

[29] R. Waelder, Equivariant elliptic genera, math.AG/0603521.

Department of Mathematics, University of Wisconsin,, Madison Wi 53706,

E-mail address: borisov@math.wisc.edu

Department of Mathematics, University of Illinois, Chicago, IL 60607

E-mail address: libgober@math.uic.edu 